\title{
The influence of the Hall effect on the global stability of cool protostellar disks
}

\author{
G. Rüdiger ${ }^{1,3}$ and L. L. Kitchatinov ${ }^{1,2}$ \\ 1 Astrophysikalisches Institut Potsdam, An der Sternwarte 16, 14482 Potsdam, Germany \\ e-mail: gruediger@aip.de \\ 2 Institute for Solar-Terrestrial Physics, PO Box 4026, Irkutsk 664033, Russian Federation \\ e-mail: kit@iszf.irk.ru \\ 3 Isaac Newton Institute for Mathematical Sciences, 20 Clarkson Rd, Cambridge, CB3 OEH, UK
}

Received 14 July 2004 / Accepted 5 January 2005

\begin{abstract}
The influence of the Hall effect on the global stability of cool Kepler disks under the influence of an axial magnetic field is considered. For sufficiently large magnetic Reynolds numbers Rm the magnetorotational instability (MRI) exists in a finite interval of magnetic field amplitudes, $B_{\min }<B<B_{\max }$. For Kepler disks the pure MRI needs both rather high Rm (representing the needed electrical conductivity) as well as $B_{\min }$ of order $0.1 \mathrm{G}$. The magnetic field pattern resulting from our global and linear calculations is of quadrupolar parity. For magnetic fields antiparallel to the rotation axis the Hall effect reduces the minimum magnetic Reynolds number by about one order of magnitude. The $B_{\min }$, however, is even (sightly) increased (see Fig. 6). For magnetic fields parallel to the rotation axis the Hall effect drives its own instability without the action of the Lorentz force. The corresponding critical magnetic Reynolds number proves to be larger with the Hall effect $(\mathrm{Rm} \sim 10)$ than without the Hall effect $(\mathrm{Rm} \sim 7)$ so that the Hall effect for parallel fields even disturbs the formation of MHD-instability in cool protoplanetary disks. If the disk is supercritical then the main result of the Hall effect for positive fields is the strong reduction of the minimum magnetic field amplitude which is necessary to start the instability. Observations must show whether in star-forming regions the rotation axis and the magnetic field orientation are correlated or are anticorrelated. If the magnetic fields are large enough then our model predicts the dominance of fields antiparallel to the rotation axis.
\end{abstract}

Key words. magnetohydrodynamics (MHD) - instabilities - magnetic fields - accretion, accretion disks

\section{Introduction}

The Hall effect in protostellar disks, with their low degree of ionization, has recently become a subject of increasing interest due to its relevance to the stability and the angular momentum transport in the disks. The Hall effect can amplify or suppress the standard magnetorotational instability (hereafter MRI, see Balbus \& Hawley 1991) depending on the sign of the product of angular velocity and magnetic field projections on the wave vector of a disturbance. The effect was found to destabilize when the product is negative (Wardle 1999; Balbus \& Terquem 2001; Rüdiger \& Shalybkov 2004). In this paper we shall present a study for the global stability of a differentially rotating disk of given (small) thickness and (low) temperature.

As the solution of the induction equation alone, the Hall effect can drive its own instability. The instability does not require rotation; it also exists for a plane shear flow. For $\mathrm{d} \Omega / \mathrm{d} R<$ 0 this shear-Hall instability develops when the axial magnetic field is positive and vice versa. Similar to MRI, it only exists between a minimum field, $B_{\min }$, and a maximum field, $B_{\max }$. For a positive magnetic field, MRI and the Hall effect amplify each other close to $B_{\min }$, and they compete close to $B_{\max }$. Both boundaries of the instability range are thus reduced by the interplay of the two effects in this case.

For negative axial fields the Hall effect and MRI amplify each other when the field strength is close to $B_{\max }$. The Hall effect is destabilizing here as it transforms the value of $B_{\max }$ to higher values. The Hall effect and MRI compete, however, close to $B_{\min }$. Here, the Hall effect is stabilizing as the $B_{\min }$ is increased. Both boundaries of the instability range are thus enhanced by the interplay of the two effects in this case.

\section{Local approximation}

\subsection{Linearized equations}

The conditions in protostellar disks were discussed by Balbus \& Terquem (2001). The disk material is partly ionized plasma where ions are well linked to neutrals but electrons are not. This leads to the induction equation including an additional term compared to the standard one-fluid MHD, i.e.

$\frac{\partial \boldsymbol{B}}{\partial t}=\nabla \times\left(\boldsymbol{u} \times \boldsymbol{B}+\boldsymbol{u}_{\mathrm{H}} \times \boldsymbol{B}-\eta \nabla \times \boldsymbol{B}\right)$ 
where the second term on the right stands for the Hall electromotive force with the effective velocity, $\boldsymbol{u}_{\mathrm{H}}$, proportional to the current density, $\boldsymbol{J}=\nabla \times \boldsymbol{B} / \mu_{0}$ :

$$
\begin{aligned}
\boldsymbol{u}_{\mathrm{H}} & =-\frac{\boldsymbol{J}}{e n_{\mathrm{e}}}=-\eta C_{\mathrm{H}} \frac{\nabla \times \boldsymbol{B}}{B}, \\
C_{\mathrm{H}} & =\frac{\omega_{\mathrm{ce}}}{v_{\mathrm{e}}} .
\end{aligned}
$$

In these equations, $n_{\mathrm{e}}$ is the electron number density, $\omega_{\mathrm{ce}}=$ $e B / \mathrm{cm}_{\mathrm{e}}$ is the cyclotron frequency, and $v_{\mathrm{e}}$ is collision frequency of electrons. The reason to introduce the "Hall velocity" (2) is that it helps in interpreting future results. Depending on the magnetic field direction the quantity $C_{\mathrm{H}}$ can be positive or negative.

The equation of motion reads

$$
\frac{\partial \boldsymbol{u}}{\partial t}+(\boldsymbol{u} \cdot \nabla) \boldsymbol{u}=-\frac{1}{\rho} \nabla P-\nabla \Phi+\frac{1}{\rho} \boldsymbol{J} \times \boldsymbol{B}+v \Delta \boldsymbol{u},
$$

where the viscosity term is kept for numerical reasons although the viscosity is small for protostellar disks. We shall see that the stability parameters do not depend on the viscosity whenever the magnetic Prandtl number,

$$
\operatorname{Pm}=\frac{v}{\eta}
$$

is below 0.1 . We assume an incompressible fluid, $\operatorname{div} \boldsymbol{u}=0$.

The reference state includes a non-uniform rotation with the angular velocity, $\Omega$, dependent on the distance $s$ to the rotation axis, and a uniform axial magnetic field $\boldsymbol{B}_{0} \| \boldsymbol{\Omega}$. Linear stability or instability against small disturbances is considered.

For a local approximation a Cartesian coordinate system rotating with the local angular velocity $\Omega$ is used with $x, y$ and $z$ pointing in the radial, azimuthal and vertical directions (see Balbus \& Hawley 1991; Brandenburg et al. 1995). The local approximation concerns perturbations whose spatial scales are small compared to the global scale of the disk parameters. The rotation law can then be approximated by the shear flow $\boldsymbol{U}_{0}=-\hat{\boldsymbol{e}}_{y} \Omega q x$ where $q$ is the (constant) local shear. The linearized MHD equations with the Hall effect read

$$
\begin{gathered}
\frac{\partial \boldsymbol{B}^{\prime}}{\partial t}-B_{0} \frac{\partial \boldsymbol{u}^{\prime}}{\partial z}-x q \Omega \frac{\partial \boldsymbol{B}^{\prime}}{\partial y}+q \Omega B_{x}^{\prime} \hat{\boldsymbol{e}}_{y} \\
-\eta \Delta \boldsymbol{B}^{\prime}+\eta C_{\mathrm{H}} \frac{\partial\left(\nabla \times \boldsymbol{B}^{\prime}\right)}{\partial z}=0, \\
\frac{\partial \boldsymbol{u}^{\prime}}{\partial t}+2 \Omega \hat{\boldsymbol{e}}_{z} \times \boldsymbol{u}^{\prime}-x q \Omega \frac{\partial \boldsymbol{u}^{\prime}}{\partial y}-q \Omega u_{x}^{\prime} \hat{\boldsymbol{e}}_{y} \\
-\frac{B_{0}}{4 \pi \rho} \frac{\partial \boldsymbol{B}^{\prime}}{\partial z}+\frac{1}{\rho} \nabla P^{\prime}-v \Delta \boldsymbol{u}^{\prime}=0
\end{gathered}
$$

with dashes indicating the small disturbances.

Considering plane waves with $\boldsymbol{B}^{\prime}, \boldsymbol{u}^{\prime}, P^{\prime} \sim \exp (\gamma t+\mathrm{i} k z)$ leads to the dispersion relation

$$
\begin{gathered}
\left(\left(\gamma+\eta k^{2}\right)^{2}+\omega_{\mathrm{H}}\left(\omega_{\mathrm{H}}-q \Omega\right)\right)\left(\left(\gamma+v k^{2}\right)^{2}+2(2-q) \Omega^{2}\right) \\
+\omega_{\mathrm{A}}^{2}\left(\omega_{\mathrm{A}}^{2}-2 q \Omega^{2}+2\left(\gamma+v k^{2}\right)\left(\gamma+\eta k^{2}\right)\right) \\
+(4-q) \omega_{\mathrm{A}}^{2} \omega_{\mathrm{H}} \Omega=0
\end{gathered}
$$

with the Alfvén and the Hall frequencies

$\omega_{\mathrm{A}}=k B_{0} / \sqrt{\mu_{0} \rho}, \quad \omega_{\mathrm{H}}=\eta k^{2} C_{\mathrm{H}}$.

If overstable modes are ignored, $\gamma=0$ in Eq. (7) gives the equation

$$
\begin{aligned}
(1+ & \left.C_{\mathrm{H}}\left(C_{\mathrm{H}}-q \mathrm{Rm}\right)\right)\left(1+2(2-q) \frac{\mathrm{Rm}^{2}}{\mathrm{Pm}^{2}}\right) \\
& +\frac{\mathrm{Ha}^{2} \mathrm{Rm}}{\mathrm{Pm}}\left((4-q) C_{\mathrm{H}}-2 q \mathrm{Rm}\right)+2 \mathrm{Ha}^{2}+\mathrm{Ha}^{4}=0,
\end{aligned}
$$

for the marginal stability separating the regions of instability $(\mathfrak{R}(\gamma)>0)$ and stability $(\mathfrak{R}(\gamma)<0)$. Here $\mathrm{Rm}$ and Ha are the local magnetic Reynolds number and the Hartmann number

$$
\mathrm{Rm}=\frac{\Omega}{\eta k^{2}}, \quad \mathrm{Ha}=\frac{\omega_{\mathrm{A}}}{k^{2} \sqrt{\eta v}},
$$

both taken as positive-definite throughout the paper. The shear is obviously necessary for any instability because Eq. (9) provides solutions with real $C_{\mathrm{H}}$ and Ha only with finite values of $q$ and $\mathrm{Rm}$.

\subsection{Shear-Hall instability}

Consider the case where the Hall frequency (8) is large,

$\omega_{\mathrm{H}}^{2} \gg \omega_{\mathrm{A}}^{2}$.

Then the dispersion relation (7) reduces to

$\left(\gamma+\eta k^{2}\right)^{2}+\omega_{\mathrm{H}}\left(\omega_{\mathrm{H}}-q \Omega\right)=0$.

The induction equation has been decoupled from the equation of motion, and the dispersion relation (12) can be found from the induction equation alone. An instability exists if the magnetic Reynolds number exceeds the minimum value $2 / q$. If $q$ is positive it exists for positive $C_{\mathrm{H}}$, i.e. for the external magnetic field parallel to the angular velocity, and vice versa $\left(q \omega_{\mathrm{H}}\right.$ must be positive, see Eq. (12)). For large $\mathrm{Rm} \gg \mathrm{Rm}_{\min }$, the instability region is given by

$\frac{1}{q \mathrm{Rm}}<C_{\mathrm{H}}<q \mathrm{Rm}$.

The Hall parameter (3) is proportional to the magnetic field. Similar to MRI, the shear-Hall instability exists in a limited range of magnetic fields. In a further similarity, the maximum growth rate is controlled by the local Oort A value, i.e.

$\gamma_{\max }+\eta k^{2}=q \Omega / 2$.

\section{3. $M R I$ for low conductivity, $P m \ll 1$}

For the small magnetic Prandtl numbers expected for protostellar disks an appropriate scaling of the MRI parameters exists. Without the Hall effect $\left(C_{\mathrm{H}}=0\right)$ Eq. (9) yields the neutral stability condition

$\mathrm{Rm}^{2}=\frac{\mathrm{Pm}^{2}\left(1+\mathrm{Ha}^{2}\right)^{2}}{2\left(q \mathrm{Pm} \mathrm{Ha}^{2}-2+q\right)}$ 
for MRI alone. Suppose that Pm is decreased by decreasing the viscosity but keeping the magnetic diffusivity finite. Then the Lundquist number

$S=\sqrt{\mathrm{Pm}} \mathrm{Ha}=\frac{\omega_{\mathrm{A}}}{\eta k^{2}}$,

remains finite. At small Pm, Eq. (15) becomes

$\mathrm{Rm}^{2}=\frac{S^{4}}{2\left(q S^{2}-2+q\right)}$.

This equation does not include Pm which means that the actual viscosity value is not important provided that it is smaller than the magnetic diffusivity. We shall see in Sect. 4 that a representation of MRI in terms of both the magnetic Reynolds and Lundquist numbers becomes independent of Pm when the latter drops below (say) 0.1. In a global model, therefore, a small but finite viscosity can be kept for numerical stability to produce the results which remain valid for arbitrarily small Pm when represented in terms of $\mathrm{Rm}$ and $S$. Hereafter, we always use the Lundquist number (16) rather than Ha.

\section{4. $M R I$ plus Hall effect for $P m=1$}

Consider the interplay of MRI and the Hall effect for the simplifying case of $\mathrm{Pm}=1$. Then the marginal stability Eq. (9) becomes

$$
\begin{aligned}
S^{2}+2 C_{\mathrm{H}} \mathrm{Rm}+1 & =\frac{q}{2} \operatorname{Rm}\left(2 \mathrm{Rm}+C_{\mathrm{H}}\right) \\
& \mp\left(2 \mathrm{Rm}-C_{\mathrm{H}}\right)\left(\frac{q^{2} \mathrm{Rm}^{2}}{4}-1\right)^{1 / 2} .
\end{aligned}
$$

The plus and minus signs on the RHS of this equation define two boundaries of the instability region for a given Rm provided that the magnetic Reynolds number exceeds the minimum value of $\mathrm{Rm}_{\min }=2 / q$.

Note that the Hall parameter (3) and the Lundquist number (16) are both linear in the external field $B_{0}$. Their ratio

$\beta=\frac{C_{\mathrm{H}}}{S}$,

therefore, characterizes mainly the material. It may be taken to be positive for magnetic fields parallel to the rotation axis and negative for magnetic fields antiparallel to the rotation axis.

For both cases the results are quite different. Consider the instability region for very large $\mathrm{Rm} \gg \max \left(\mathrm{Rm}_{\min }, \beta^{-1}\right)$. For positive $\beta$ it is

$\frac{1}{\beta q \mathrm{Rm}}<S<\mathrm{Rm}\left(\left(2 q+\beta^{2}\right)^{1 / 2}-\beta\right)$.

The lower limit is the same as in Eq. (13). $B_{\min }$ for positive $\beta$ is thus controlled by the shear-Hall instability. The lower bound is small compared to $S_{\text {min }}=\sqrt{(2-q) / q}$ expected from Eq. (17) for MRI. The Hall effect for positive $B_{0}$ amplifies MRI for the fields close to $B_{\min }$. The effect is, however, stabilizing close to $B_{\max }$ because the upper limit in Eq. (20) decreases with $\beta$.

The instability domain for negative $\beta$ is

$|\beta|(2-q) \mathrm{Rm}<S<\left(\sqrt{2 q+\beta^{2}}+|\beta|\right) \mathrm{Rm}$.

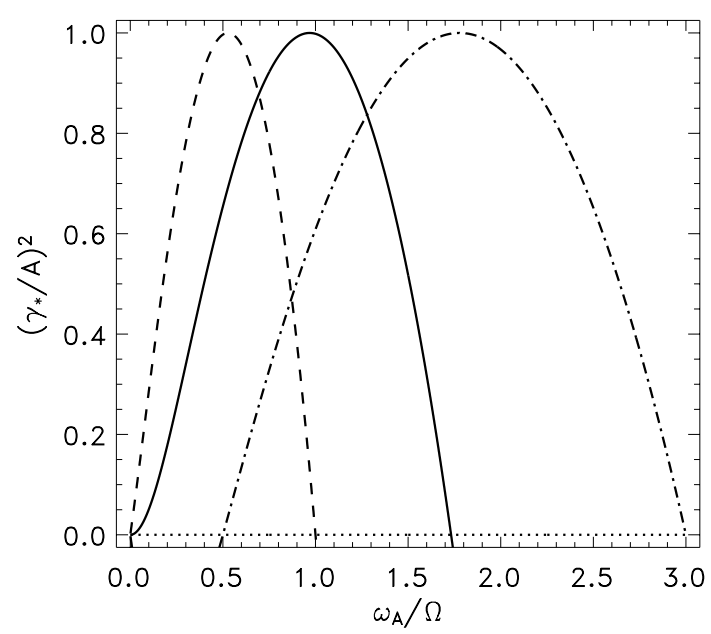

Fig. 1. The growth rates, $\gamma_{*}=\gamma+\eta k^{2}$ in units of $A=q \Omega / 2$ as functions of the normalized field strength for MRI alone (full line) and MRI modified by the Hall effect of positive fields ( $\beta=1$, dashed line) and negative fields ( $\beta=-1$, dashed-dotted). $\mathrm{Pm}=1$.

Both bounds increase with $|\beta|$. Here the Hall effect is thus destabilizing close to $B_{\max }$ (Wardle 1999; Balbus \& Terquem 2001) and stabilizing close to $B_{\min }$.

In Fig. 1 the interaction of MRI with the Hall effect is demonstrated by the growth rates derived from the dispersion relation (7) for Pm $=1$. The solid line gives the typical growth rate profile for the MRI alone. For positive fields the profile is moved by the Hall effect to the left and for negative fields the profile is moved to the right. At the strong-field limits only the negative Hall effect is thus destabilizing. In opposition to that the positive Hall effect even disturbes the instability and may not help to produce the desired turbulence in cool disks.

\section{A global model}

The above results for growth or decay of small disturbances taken from the local dispersion relation have been known since the papers of Wardle (1999) and Balbus \& Terquem (2001). Contrary to the local considerations we shall present in the following the results for global Kepler flow models with Ohmic dissipation and the Hall effect included. The progress of such an approach is to find exact values for the critical Reynolds numbers, the minimal and maximal magnetic amplitudes and the global geometry of the resulting instabilty pattern. As the limiting magnetic fields prove to be surprisingly high (and rather sensitive to the Hall effect) information about the minimum magnetic fields is important for the discussion of the (magneto-)hydrodynamics of cool protostellar disks.

Our global model differs from that of Kitchatinov \& Mazur (1997) only by the inclusion of the Hall effect. The model concerns a rotating disk of constant thickness, $2 H$, threaded by a uniform axial magnetic field. The rotation axis is normal to the disk, and the angular velocity, $\Omega$, depends on the distance, $R$, to the axis. This dependence is parameterized by

$\Omega(R)=\Omega_{0} \tilde{\Omega}(R)$ 
with

$\tilde{\Omega}(R)=\left(1+\left(\frac{R}{R_{0}}\right)^{3 n / 2}\right)^{-1 / n}$.

This profile describes almost uniform rotation at small distances $R \ll R_{0}$, which smoothly transforms to the Keplerian law, $\Omega \simeq \Omega_{0}\left(R_{0} / R\right)^{3 / 2}$, for large distances $R \gg R_{0}$. We use $n=2$ in Eq. (23) and $R_{0} / H=5$ for the aspect ratio and $\operatorname{div} \boldsymbol{u}=0$ as above. The pressure is excluded by curling Eq. (4). This yields for the vorticity, $\omega=\nabla \times \boldsymbol{u}$, the relation

$\frac{\partial \omega}{\partial t}=\boldsymbol{\nabla} \times(\boldsymbol{u} \times \boldsymbol{\omega}+\boldsymbol{J} \times \boldsymbol{B} / \rho)+v \Delta \boldsymbol{\omega}$.

The Eqs. (1) and (24) are about the rotation (22) and the uniform axial field, $\boldsymbol{B}_{0}=B_{0} \hat{\boldsymbol{e}}_{z}\left(\hat{\boldsymbol{e}}_{z}\right.$ is the unit vector along the rotation axis), and the normalized variables

$\hat{\boldsymbol{b}}=\boldsymbol{B}^{\prime} / B_{0}, \hat{\boldsymbol{j}}=\frac{\mu_{0} H}{B_{0}} \boldsymbol{J}^{\prime}, \hat{\boldsymbol{u}}=\boldsymbol{u}^{\prime} /\left(H \Omega_{0}\right), \hat{\boldsymbol{\omega}}=\omega^{\prime} / \Omega_{0}$,

for the disturbances are introduced. This leads to four basic dimensionless parameters among which the Hall parameter, $C_{\mathrm{H}}$, and magnetic Prandtl number, Pm, are defined by Eqs. (3) and (5) but magnetic Reynolds number and the Lundquist number now are

$\mathrm{Rm}=\frac{\Omega_{0} H^{2}}{\eta}, \quad S=\frac{B_{0} H}{\sqrt{\mu_{0} \rho} \eta}$.

These parameters control the equation system for the normalized disturbances

$$
\begin{aligned}
\frac{\partial \hat{\boldsymbol{b}}}{\partial t}= & \operatorname{Rm} \nabla \times\left(R \tilde{\Omega}(R) \hat{\boldsymbol{e}}_{\phi} \times \hat{\boldsymbol{b}}-\hat{\boldsymbol{e}}_{z} \times \hat{\boldsymbol{u}}\right)-C_{\mathrm{H}}\left(\hat{\boldsymbol{e}}_{z} \cdot \nabla\right) \hat{\boldsymbol{j}}+\Delta \hat{\boldsymbol{b}} \\
\frac{\partial \hat{\boldsymbol{\omega}}}{\partial t}= & \operatorname{Rm} \nabla \times\left(R \tilde{\Omega}(R) \hat{\boldsymbol{e}}_{\phi} \times \hat{\boldsymbol{\omega}}-\frac{\kappa^{2}}{2 \tilde{\Omega}(R)} \hat{\boldsymbol{e}}_{z} \times \hat{\boldsymbol{u}}\right) \\
& +\frac{S^{2}}{\operatorname{Rm}}\left(\hat{\boldsymbol{e}}_{z} \cdot \nabla\right) \hat{\boldsymbol{j}}+\operatorname{Pm} \Delta \hat{\boldsymbol{\omega}},
\end{aligned}
$$

where $\kappa$ is the normalized epicycle frequency

$$
\kappa^{2}=\frac{2 \tilde{\Omega}}{R} \frac{\mathrm{d}\left(R^{2} \tilde{\Omega}\right)}{\mathrm{d} R},
$$

and time and distances are normalized to the diffusion time, $H^{2} / \eta$, and the disk half-thickness, $H$. Here only the stability of the rotation law is considered, the accretion flow which is connected to this nonuniform rotation via the viscosity is neglected (see Kersalé et al. 2004).

The boundary conditions on the disk surfaces are (i) stressfree for the flow and; (ii) pseudo-vacuum conditions for the magnetic field fluctuations, i.e.

$\hat{\boldsymbol{e}}_{z} \times \hat{\boldsymbol{b}}=0$.

The solutions are required to be regular on the rotation axis and to vanish at infinity.

The linear stability analysis with $\hat{\boldsymbol{b}}, \hat{\boldsymbol{\omega}} \sim \exp (\gamma t)$ leads to an eigenvalue problem for the equation set (27) which has been solved numerically. A new variable, $y$,

$y=\frac{R / R_{0}}{1+R / R_{0}}, \quad 0 \leq y \leq 1$,

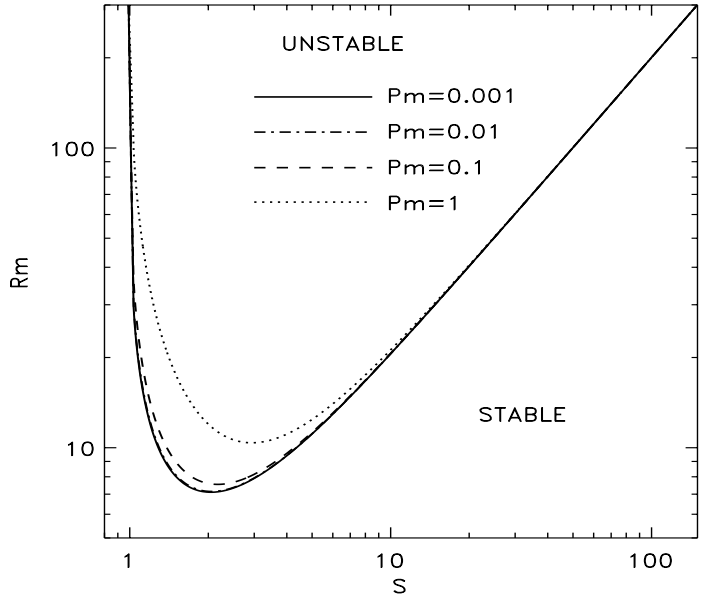

Fig. 2. Neutral stability lines of the MRI for various magnetic Prandtl numbers $\left(C_{\mathrm{H}}=0\right)$. For $\mathrm{Pm}<0.1$ the lines are almost independent of Pm.

has been introduced transforming the infinite disk to a finite domain. $R_{0}$ is the turnover radius in Eq. (23). A uniform grid in $y$ was applied which corresponds to a non-uniform grid in $R$. The eigenvalues and eigenvectors are computed by the inverse iteration method.

The system (27) allows two types of solutions with different symmetries about the disk midplane. One of the symmetry types combines a symmetric magnetic field with an antisymmetric flow field. The notation, $\mathrm{S} m$, will be used for this type of eigenmodes, where " $m$ " is the azimuthal wave number, i.e. $\mathrm{S} 0$ represents an axisymmetric mode, $\mathrm{S} 1$ defines the nonaxisymmetric mode with $m=1$, and so on. The other symmetry type combines antisymmetric magnetic field with symmetric flow. The notation $\mathrm{A} m$ is used for the eigenmodes of this type of symmetry.

The primary goal of the linear theory is to define the stability boundary in parameter space which separates the region of stable perturbations with negative or zero real part of $\gamma$ from the instability region with (exponentially) growing perturbations. The stability map strongly depends on the symmetry type of the excitation.

\section{Results}

\subsection{MRI for low conductivity (small Pm)}

Consider first with $C_{\mathrm{H}}=0$ the MRI alone. Figure 2 shows the neutral stability lines for Prandtl numbers decreasing from $\mathrm{Pm}=1$ to smaller values. The stability is almost independent of the magnetic Prandtl number for sufficiently small Pm, say $\mathrm{Pm}<0.1$. The MRI characteristics computed with moderately small $\mathrm{Pm}$ remain valid for arbitrary small $\mathrm{Pm}$. This finding may be important for predicting MRI parameters for laboratory experiments and protostellar disks where magnetic Prandtl numbers are very small. Hereafter, we fix Pm $=0.01$.

The minimum value for $\mathrm{Rm}$ for instability in the small Pm regime is

$\mathrm{Rm}_{\min } \simeq 7.1$. 


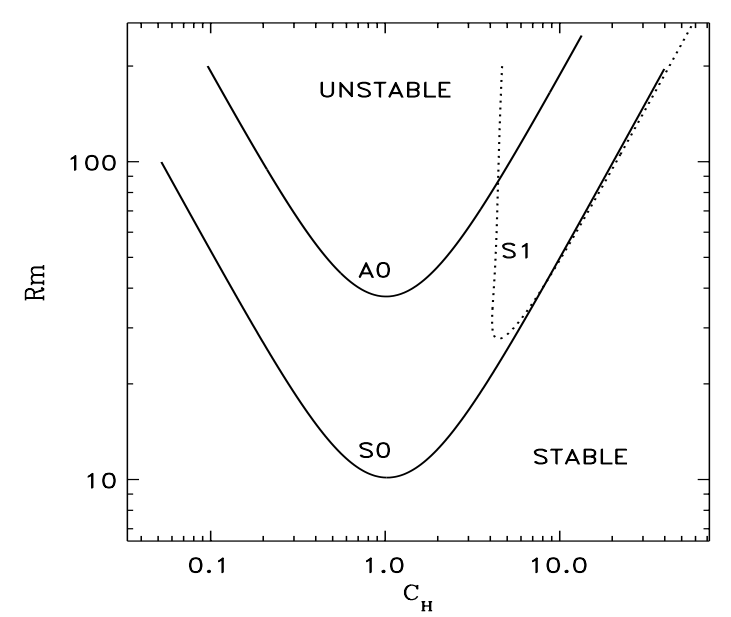

Fig. 3. Stability diagram for the shear-Hall instability (positive magnetic field, i.e. parallel to the rotation axis; only the induction equation is solved). Lines are labeled by their symmetry types. The axisymmetric quadrupolar mode (S0) is preferred.

For Reynolds numbers large compared to this value the instability exists within the magnetic range

$1<S<0.5 \cdot \mathrm{Rm}$.

Cool protostellar disks may not reach such values of the magnetic Reynolds number. On the other hand, for protostellar disks the minimum condition for the magnetic field $(S \simeq 1)$ proves to be very stringent (in great contrast to the MRI in galaxies, see Kitchatinov \& Rüdiger 2004). With the values given below for $\eta\left(10^{15} \mathrm{~cm}^{2} / \mathrm{s}\right)$, density $\left(10^{-10} \mathrm{~g} / \mathrm{cm}^{3}\right)$ and for a disk height of $0.1 \mathrm{AU}$ a minimum field of almost $0.1 \mathrm{G}$ is needed to fulfill $S=1$. It is thus tempting for different reasons to probe the shear-Hall instability for protostellar disks which, however, only exists for magnetic fields parallel to the rotation axis.

\subsection{Shear-Hall instability}

Another extreme exists for magnetic fields parallel to the rotation axis. The Hall effect for one sign of the magnetic field in connection with differential rotation can form its own instability as shown by the solution of the induction equation alone. In the present model this shear-Hall instability can be found for small Lundquist number, $S \ll C_{\mathrm{H}}$, i.e. large $\beta$ of Eq. (19). In this case the Lorentz force in (4) can be neglected so that MRI is excluded.

Figure 3 shows for $\beta \rightarrow \infty$ the stability map for modes of three basic symmetry types, ie. S0, S1, and A0. The quadrupolar axisymmetric modes $\mathrm{S} 0$ are preferentially excited. The neutral stability lines for the other symmetry types lie completely inside the unstable region of S0. It is one of the advantages of our approach that the equatorial symmetry results from the computations rather than needing to be prescribed. Salmeron \& Wardle (2003) in their extensive analysis of the vertical structure of accretion disks used those symmetry conditions at the equator valid only for A0-fields. When the system becomes unstable against A0-disturbances it is thus already unstable against S0-perturbations.

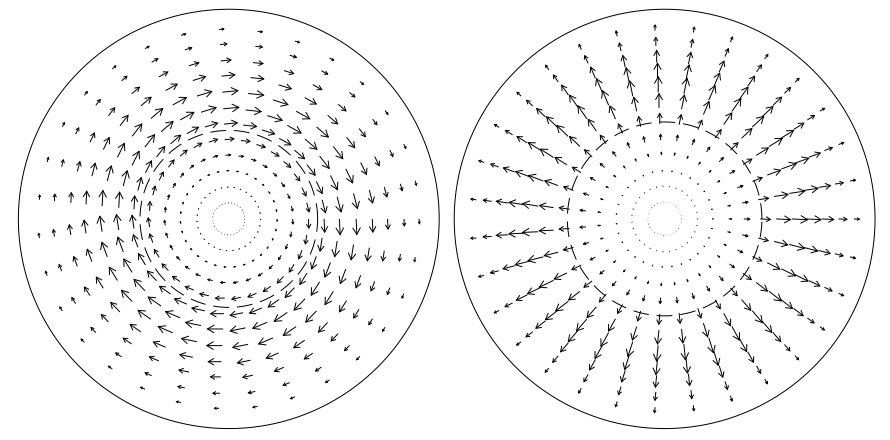

Fig. 4. Magnetic field vectors in the disk midplane for marginally stable $\mathrm{S} 0$-modes at $\mathrm{Rm}=100$ for the minimum (left) and maximum (right) Lundquist numbers, resp. The broken circle shows the turnover radius $R_{0}$ of the rotation law.

The minimum Reynolds number for all the modes is

$\mathrm{Rm}_{\min } \simeq 10$.

For large Rm, the left branch of the neutral stability line for S0-modes approaches the relation

$C_{\mathrm{H}} \simeq 5 / \mathrm{Rm}$

while the right branch is close to

$C_{\mathrm{H}} \simeq 0.2 \cdot \mathrm{Rm}$.

Note that for large magnetic Reynolds number Rm the minimum $C_{\mathrm{H}}$ necessary for instability becomes infinitely small.

The maximum fields allowing for the axisymmetric S0-modes and the nonaxisymmetric S1-modes in Fig. 3 are almost equal. This property might be important for the dynamo theory with respect to the Cowling theorem.

Equations (33)-(35) correspond to Eq. (13) obtained with the local analysis. Quantitative differences are partly due to the difference in definitions of Rm between local and global calculations. The differences are actually quite small if the transformation rule $k \rightarrow \pi / H$ between local and global formulations is used.

Figure 4 shows that the pitch angle for marginally stable disturbances is indeed very small for the minimum field producing the instability. The angle increases to about $\pi / 2$ for the maximum field. This tendency for the pitch angle can also explain the results for nonaxisymmetric disturbances. For pitch angle close to $\pi / 2$ the azimuthal structure is not significant. Accordingly, the maximum fields for the S1 and S0 modes are roughly the same (Fig. 3). Small pitch angle, however, would mean a tight winding with a small radial scale for S1 modes whose instability is then precluded by diffusion. This is why the instability region of $\mathrm{S} 1$ modes in Fig. 3 exhibits such a sharp and almost vertical boundary on the weak-field side.

The orientation of the magnetic field in relation to the rotation axis plays an important role in the interplay of differential rotation and the Hall effect. Now the two cases of parallel and antiparallel magnetic fields are considered solving the complete equations. We find that the MRI of the Kepler flow is very differently modified by the Hall effect. 


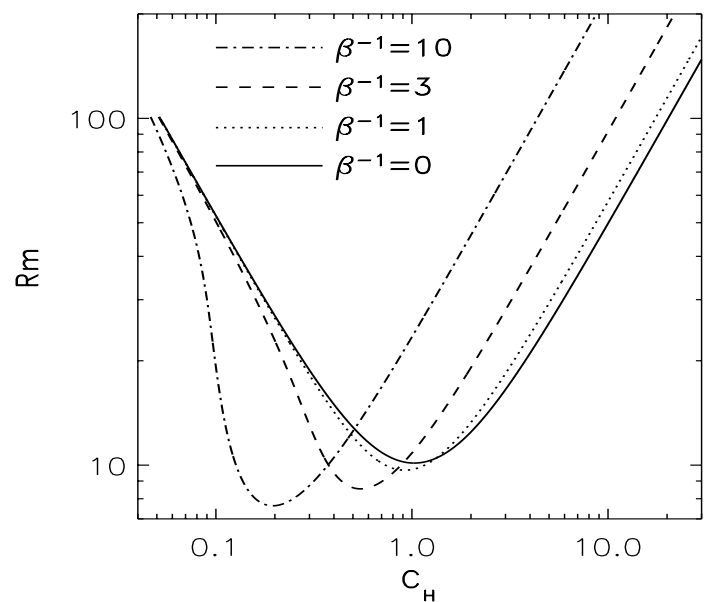

Fig. 5. The same as in Fig. 3 but for the full equation system. The Hall effect increases the minimum Rm. The solid curve for $\beta \rightarrow \infty$ is identical to the lower curve in Fig. 3. For increasing $\mathrm{Rm}$ the lower limit for the magnetic field becomes smaller and smaller.

\subsection{Positive (parallel) fields}

For positive $\beta$ the solution is located between the two realizations given in Figs. 2 and 3. The minimum magnetic Reynolds number moves from the value 7 for MRI to about 10 for the shear-Hall instability. The Hall effect does thus not support the instability of the cool Kepler flow; for all positive $\beta$ the minimum remains between 7 and 10 (Fig. 5).

Figure 5 also shows that the minimum of the possible magnetic fields is fixed by the Hall effect. We find $\mathrm{Rm} \sim 1 /(\beta \cdot S)$ so that $S \sim 1 /(\beta \cdot \mathrm{Rm})$ which for large $\mathrm{Rm}$ is smaller by orders of magnitude than $S \sim 1$ taken from Fig. 6 for negative $\beta$.

Our main interest also concerns the results for low electrical conductivity, i.e. for small Rm. The minimum Rm moves in Fig. 5 from 7.1 to 10, i.e. it increases opposite to the desired trend. If the results for parallel and antiparallel magnetic fields are compared, one of the differences is the opposite trend for the minimum magnetic Reynolds number which only for negative fields (antiparallel to the rotation axis) is reduced by the Hall effect. It is also important that for large enough $\mathrm{Rm}$ the minimum magnetic fields for the instability strongly differ for both the magnetic orientations.

\subsection{Negative (antiparallel) fields}

Figure 6 shows the stability diagram for negative $B_{0}$ for which the shear-Hall instability does not exist. The Hall effect, however, increases both instability limits of the field amplitudes. The Hall effect thus destabilizes for strong fields close to $B_{\max }$ and stabilizes close to $B_{\min }$.

On the other hand, the absolute minimum of the magnetic Reynolds number Rm is reduced. The necessary electrical conductivity of the gas is (slightly) reduced by the Hall effect. From numerical arguements, we can present only the results for a small Hall parameter $\beta$ which is still too small by one order of magnitude (see Eq. (40) below). A massive reduction of the critical magnetic Reynolds number (i.e. the necessary electrical conductivity) is thus expected from the Hall effect for

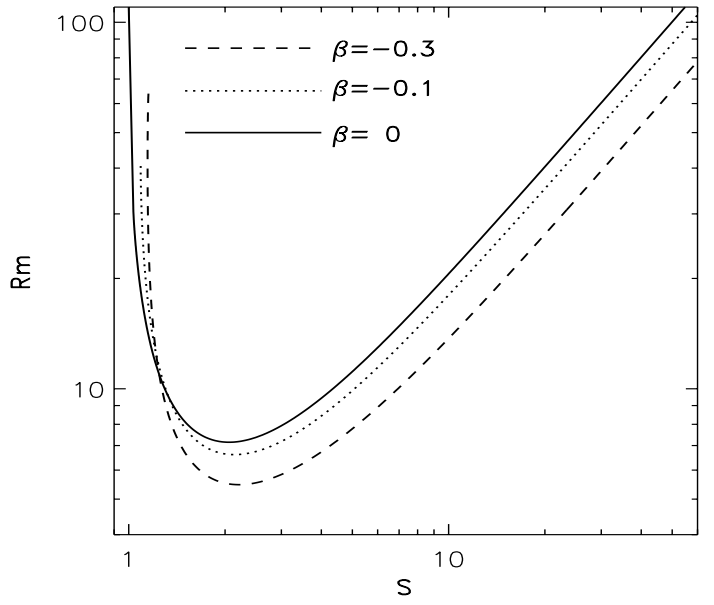

Fig. 6. Stability diagram for MRI modified by the Hall effect for magnetic fields antiparallel to the angular velocity. Note that the Hall parameter $\beta$ for real cool protostellar disks is of order unity. The Hall effect shifts the instability interval to larger Lundquist numbers, i.e. to higher magnetic fields.

Table 1. Ratio of the global magnetic to kinetic energy at minimum magnetic Reynolds number for a sequence of the Hall parameter $\beta$.

\begin{tabular}{c|llllll}
\hline \hline$\beta$ & -0.3 & -0.1 & 0 & 0.1 & 0.33 & 1 \\
$E_{\mathrm{mag}} / E_{\mathrm{kin}}$ & 0.77 & 1.20 & 1.52 & 1.94 & 3.32 & 11.4 \\
\hline
\end{tabular}

antiparallel magnetic fields. It seems indeed to be possible to use the Hall effect to realize the MRI also for the rather low electrical conductivity of cool disks.

\subsection{Energy relation and angular momentum transport}

Linear computations cannot provide energy values. It is possible, however, to compare their global magnetic and kinetic energies. These ratios of the total (volume integrated) energies are given in Table 1 for a sequence of values of the Hall parameter $\beta$. The total energy is normally dominated by its magnetic part in agreement with other simulations of MRI (cf. Stone \& Norman 1994; Brandenburg et al. 1995; Sano \& Stone 2002). The energy ratio which we obtain is, however, decreasing and even drops below unity when the Hall parameter becomes more negative. A similar trend can also be found in the simulation results of Sano \& Stone (2002). The angular momentum always flows outwards, and it is dominated by the Maxwell stress (Balbus \& Hawley 1991; Brandenburg et al. 1996). The relative contribution of the Reynolds stress also increases with decreasing $\beta$, in accordance with the results of Sano \& Stone (2002, their Table 4).

\section{Discussion}

Ionization of protostellar disks material should be extremely low (Gammie 1996). The electrical conductivity is then controlled by electron-neutral collisions and the magnetic diffusivity

$\eta=234 \frac{n}{n_{\mathrm{e}}} T^{1 / 2} \mathrm{~cm}^{2} \mathrm{~s}^{-1}$ 
is inversely proportionate to the ionization ratio (cf. Balbus \& Terquem 2001) where $n_{\mathrm{e}}$ and $n$ are the number densities of electrons and neutrals. Then the magnetic Reynolds number (26) can be estimated with Eq. (36) as

$\mathrm{Rm}=2 \times 10^{15}\left(\frac{n_{\mathrm{e}}}{n}\right)\left(\frac{T}{100 \mathrm{~K}}\right)^{-1 / 2}\left(\frac{\tau_{\mathrm{rot}}}{0.1 \mathrm{yr}}\right)^{-1}\left(\frac{H}{0.1 \mathrm{AU}}\right)^{2}$,

where $\tau_{\text {rot }}$ is the rotation period at the distance $s_{0}=5 H$.

The ionization fraction, $n_{\mathrm{e}} / n$, is very uncertain. We estimate first its value required for instability. Without the Hall effect our results suggest $\mathrm{Rm} \simeq 10$ for instability. Assuming numerical values in Eq. (37) as representative, one finds

$\frac{n_{\mathrm{e}}}{n} \simeq 10^{-14}$

as the critical value. Even such a low ionization is problematic for protostellar disks (Stepinski 1992; Gammie 1996). Collisional ionization is inefficient for $T<10^{3} \mathrm{~K}$. The cosmic ray, however, can provide ionization fractions of $n_{\mathrm{e}} / n \simeq$ $10^{-12}$ if the column density $\Sigma$ does not exceed $10^{2} \mathrm{~g} \mathrm{~cm}^{-2}$ (Umebayashi \& Nakano 1981; Gammie 1996). With this value, Eq. (37) for thick disks $(H \simeq 0.1 \mathrm{AU})$ provides $\mathrm{Rm} \simeq 10^{3}$ but this value reduces to 10 for thinner disks $(H \simeq 0.01 \mathrm{AU})$.

The Hall parameter (3) can be estimated as

$C_{\mathrm{H}} \simeq 20\left(\frac{T}{100 \mathrm{~K}}\right)^{-1 / 2}\left(\frac{n}{10^{14} \mathrm{~cm}^{-3}}\right)^{-1} \frac{B}{1 G}$,

and $\beta$ reads $\beta$ of Eq. (19), reads

$|\beta| \simeq 2 \times 10^{-12} \frac{n}{n_{\mathrm{e}}}\left(\frac{n}{10^{14} \mathrm{~cm}^{-3}}\right)^{-1 / 2}\left(\frac{H}{0.1 \mathrm{AU}}\right)^{-1}$.

With a particle density of $n \simeq 10^{14} \mathrm{~cm}^{-3}$, this relation leads to the value of

$|\beta| \simeq 2$,

so that the Hall effect should indeed be very important. With such large values one can take from Fig. 5 that the pure shearHall instability dominates for positive fields. In Fig. 7 the numerical results are summarized for the dependence of the critical magnetic Reynolds number on the Hall parameter $\beta$. For negative $\beta$ of order unity it is obvious that the critical magnetic Reynolds number is reduced by at least one order of magnitude by the Hall effect.

The boundaries of the instability domain also strongly depend on the field orientation. Only for antiparallel fields does an instability exist for $\mathrm{Rm}<7$. If the magnetic Reynolds number exceeds $\mathrm{O}(10)$ then both magnetic orientations lead to instability but for rather different magnetic amplitudes. It is

$0.1 \mathrm{G}<B_{0}<10 \mathrm{G}$

for antiparallel fields and

$0.001 \mathrm{G}<B_{0}<1 \mathrm{G}$

for parallel fields, both taken for $\mathrm{Rm}=100$. Note that the magnetic fields of meteorites vary between $1 \mathrm{G}$ and $10 \mathrm{G}$, close to the upper limits of the above equations. This coincidence suggests that the instability may drive a dynamo which saturates

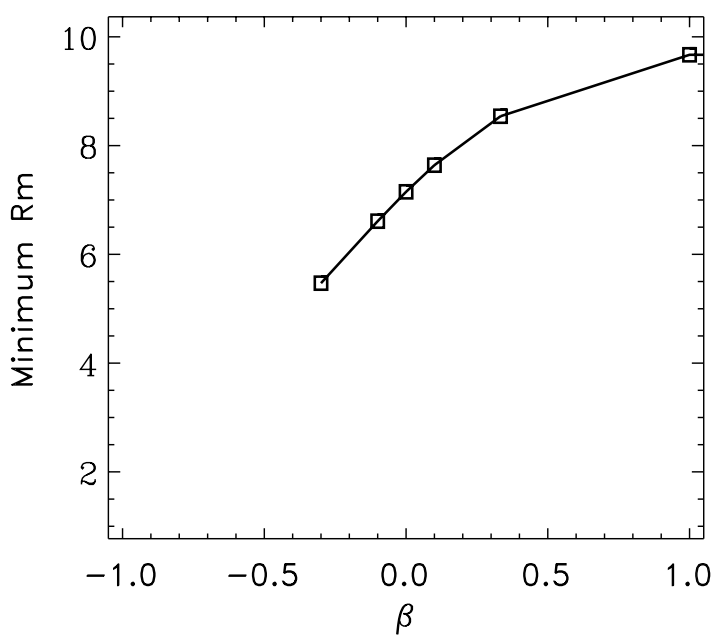

Fig. 7. The computed minimum magnetic Reynolds number as a function of the Hall parameter. $\beta=0$ denotes MRI. The results for $\beta<-0.3$ are not yet known.

when the field is amplified to the upper boundary of the instability interval. Also, the minimum magnetic fields allowing the instability prove to be rather strong. If the external magnetic fields never exceed $0.1 \mathrm{G}$ in amplitude then only the Hall effect of parallel fields would lead to the instability necessary to remove the angular momentum in the Kepler disk but then the ionization must be high enough. If it is not, then the Hall effect for antiparallel fields is needed but the magnetic field must exceed $0.1 \mathrm{G}$ in this case. It this clear from such considerations that in dense and cold globules of molecular clouds there are severe limitations to the necessary transport of angular momentum by magnetic instabilities.

Acknowledgements. L.L.K. is grateful to the Astrophysical Institute Potsdam for its hospitality and the visitor support.

\section{References}

Balbus, S. F., \& Hawley, J. F. 1991, ApJ, 376, 214

Balbus, S. F., \& Terquem, C. 2001, ApJ, 552, 235

Brandenburg, A., Nordlund, Å., Stein, R. F., \& Torkelsson, U. 1995, ApJ, 446, 741

Brandenburg, A., Nordlund, Å., Stein, R. F., \& Torkelsson, U. 1996, ApJ, 458, L45

Gammie, C. F. 1996, ApJ, 457, 355

Kersalé, E., Hughes, D. W., Ogilvie, G. I., Tobias, S. M., \& Weiss, N. O. 2004, ApJ, 602, 892

Kitchatinov, L. L., \& Mazur, M. V. 1997, A\&A, 324, 821

Kitchatinov, L. L., \& Rüdiger, G. 2004, A\&A, 424, 565

Rüdiger, G., \& Shalybkov, D. 2004, Phys. Rev. E, 69, 016303

Salmeron, R., \& Wardle, M. 2003, MNRAS, 345, 992

Sano, T., \& Stone, J. M. 2002, ApJ, 557, 534

Stepinski, T. F. 1992, Icarus, 97, 130

Stone, J. M., \& Norman, M. L. 1994, ApJ, 433, 746

Umebayashi, T., \& Nakano, T. 1981, PASJ, 33, 617

Wardle, M. 1999, MNRAS, 307, 849 